\title{
Ciclo reproductivo del pargo lunarejo Lutjanus guttatus (Steindachner, 1869) en las costas de Guerrero, México
}

\author{
Reproductive cycle of the spotted rose snapper Lutjanus guttatus (Steindachner, 1869) in the \\ Guerrero coast, Mexico
}

\author{
Marcial Arellano-Martínez ${ }^{1}$, Agustín Rojas-Herrera ${ }^{2}$, Federico García-Domínguez ${ }^{1}$, Bertha P. \\ Ceballos-Vázquez ${ }^{1}$ y Marcial Villalejo-Fuerte ${ }^{1}$ \\ ${ }^{1}$ Centro Interdisciplinario de Ciencias Marinas. Instituto Politécnico Nacional. Apdo. Postal 592, La Paz, B.C.S. 23000, México \\ ${ }^{2}$ Escuela Superior de Ecología Marina. Acapulco, Guerrero, México. \\ marellam@redipn.ipn.mx
}

Resumen.- Se estableció el ciclo reproductivo del pargo lunarejo Lutjanus guttatus, un pez de gran importancia comercial. Los organismos se recolectaron mensualmente desde febrero de 1993 a diciembre de 1994. La proporción de sexos poblacional fue de $1 \mathrm{M}: 0.96 \mathrm{H}$ y no se encontró una diferencia significativa $(\mathrm{P} \leq 0,05)$ de la proporción esperada de 1:1. Las gónadas se analizaron histológicamente y se describieron las fases de desarrollo gonadal. L. guttatus presenta un desarrollo asincrónico de las gónadas y es un desovador parcial. La actividad reproductiva estuvo presente todo el año aunque con dos períodos reproductivos principales (marzo-abril y agosto-noviembre) representados por altos porcentajes de gónadas categorizadas como maduras y en desove. Estos períodos coinciden con valores elevados del índice gonadosomático (IGS) y valores bajos del índice hepatosomático (IH). El factor de condición (FC) presentó una tendencia similar a la del IGS con los máximos valores durante la época de reproducción y disminuyendo hacia las épocas de reposo. Se considera que el IGS representa adecuadamente la actividad reproductiva y que puede haber una relación inversa entre la actividad reproductiva y el IH.

Palabras clave: Biología reproductiva, Lutjanus guttatus, índice gonadosomático, Guerrero, México.

\section{Introducción}

El pargo lunarejo Lutjanus guttatus (Steindachner, 1869) es una especie demersal, que habita en arrecifes costeros, hasta unos $30 \mathrm{~m}$ de profundidad, generalmente solitario o en pequeños grupos, pero ocasionalmente forma grandes cardúmenes; los juveniles viven en estuarios y bocas de río (Allen 1995). Esta especie se distribuye desde el Golfo de California, México hasta Perú (Allen 1985). Las capturas comerciales más importantes de las pesquerías ribereñas de peces, en el estado de Guerrero, México, se basan principalmente en las familias Carangidae, Haemulidae, Ariidae y Lutjanidae. De esta última destacan por su volumen de

\begin{abstract}
The reproductive cycle of the spotted rose snapper Lutjanus guttatus, a fish of great commercial importance was established. The organisms were sampled monthly from February 1993 to December 1994. The population sex ratio was $1 \mathrm{M}: 0.96 \mathrm{~F}$ and it was not a significant difference $(\mathrm{P} \leq 0.05)$ from the prospective proportion of $1: 1$. The gonads were histologicaly analyzed and the phases of gonadal development were described. $L$. guttatus presents an asynchronous development of the gonads and it is a partial spawner. The reproductive activity was present around the year although with two main reproductive periods (MarchApril and August-November) represented by high percentages of mature and spawning staged gonads. These high percentages coincide with high values of the gonadosomatic index (GSI) and with low values of the hepatosomatic index (HI). The condition factor (CF) presented a similar tendency to IGS, with high values during the reproductive period and diminishing toward the resting period. It is considered that the GSI represents the reproductive activity appropriately and that it have an inverse relationship between the reproductive activity and the IH.
\end{abstract}

Keywords: Reproductive biology, Lutjanus guttatus, gonadosomatic index, Guerrero, Mexico.

captura y valor comercial las especies Lutjanus peru (1000 tm al año aproximadamente) y L. guttatus (650 tm al año aproximadamente) (Santamaría 1998). Así, el pargo lunarejo constituye un importante componente de la pesquería artesanal en la zona de estudio.

A pesar de su importancia comercial, poco se sabe acerca de la biología reproductiva de L. guttatus. Rojas (1997) reporta que L. guttatus en el Golfo de Nicoya, Costa Rica, presenta un prolongado período de desove, con dos picos principales, uno en el mes de abril y otro más en el mes de octubre. Cruz-Romero et al. (1988) determinaron dos picos reproductivos para esta especie en el estado de Colima, México, uno durante la época de 
lluvias que coincide con la presencia de temperaturas altas (julio-noviembre) y otro más corto de febrero a abril.

También se ha demostrado un prolongado período de desove para otras especies de la familia Lutjanidae, tanto en el Océano Pacífico como en el Océano Atlántico (Munro et al. 1973, Thomson \& Munro 1973, Brouard \& GranPerrin 1984, Cruz-Romero et al. 1991).

El objetivo del presente estudio fue determinar el ciclo reproductivo y época de reproducción del pargo lunarejo L. guttatus en las costas del estado de Guerrero, México.

\section{Materiales y Métodos}

El área de estudio se localiza frente a las costas del estado de Guerrero, México, entre los $17^{\circ} 20^{\prime}$ y $17^{\circ} 32^{\prime}$ de latitud norte y los $99^{\circ} 08^{\prime}$ y $99^{\circ} 43^{\prime}$ de longitud oeste (Fig. 1). Se hicieron recolectas mensuales al azar de especímenes adultos de $L$. guttatus por parte de personal de la Facultad de Ecología Marina del estado de Guerrero, desde febrero de 1993 a diciembre de 1994, excepto en mayo de 1993 y en septiembre de 1994, a partir de las capturas comerciales que se realizan en el poblado de Punta Maldonado, Guerrero, México. Dichas capturas se realizan durante la noche, con líneas de anzuelo (No. 6, 7 y 8), a una profundidad de entre 10 y $30 \mathrm{~m}$. La carnada que se utilizó es calamar o sardina dependiendo de la disponibilidad. Se registró la longitud total (cm) y el peso total (g) de cada ejemplar y se extrajeron la gónada y el hígado y se registraron los pesos correspondientes; las gónadas se fijaron en formalina al 10\%. En febrero, junio y septiembre de 1993 las gónadas no fueron fijadas correctamente por lo que no se pudo hacer el estudio histológico.

Posteriormente se tomó una sección de cada gónada y se les aplicó la técnica histológica convencional (Humanson 1979) que consiste en deshidratación en una serie de alcoholes en concentración creciente e inclusión en parafina. Los cortes se hicieron a 7 micrómetros, y se tiñeron con hematoxilina y se contrastaron con eosina.

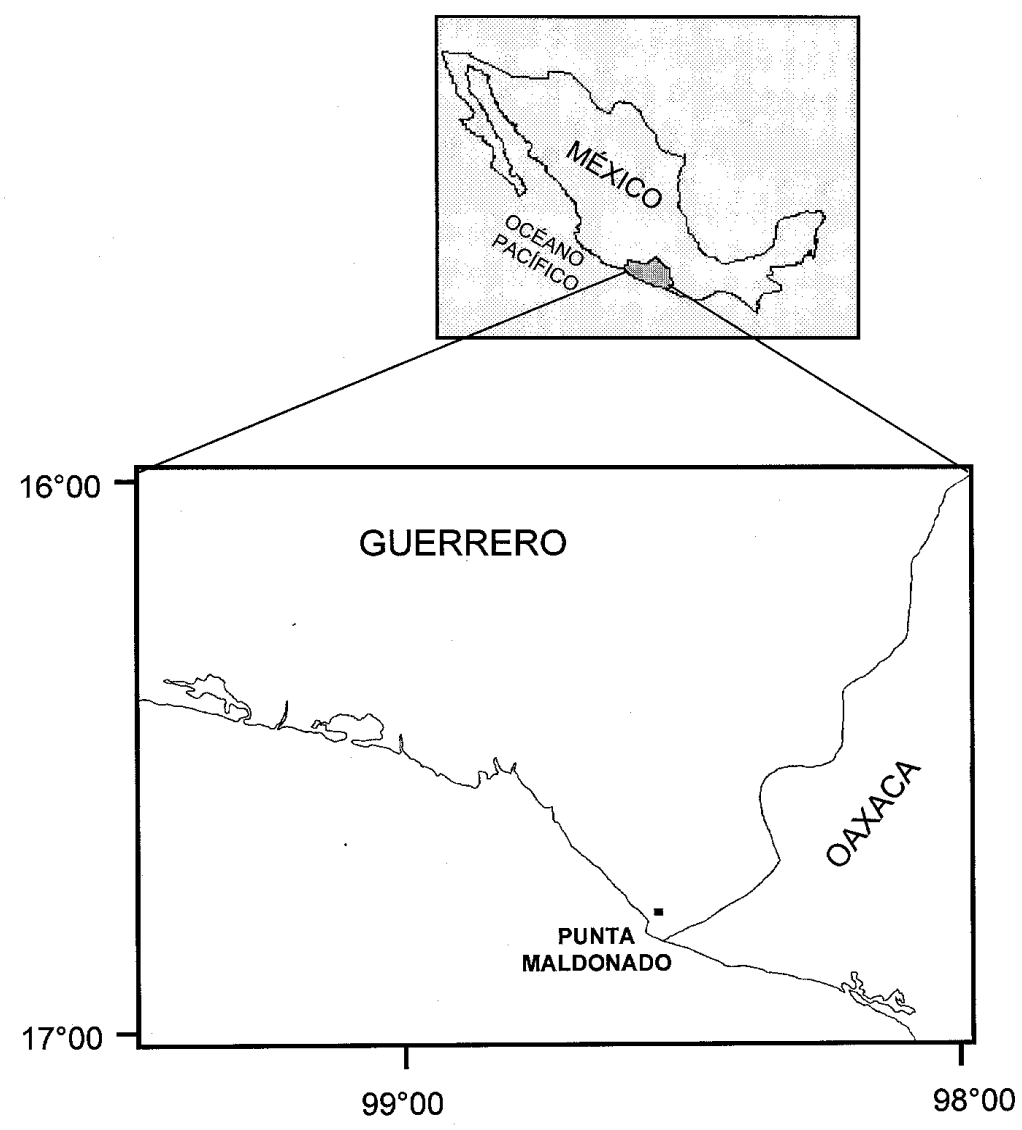

Figura 1

Localización del área de muestreo. 
Se tomaron como base los 5 estadios de desarrollo gonadal (inmadura, en desarrollo, desarrollada, madura y desove) establecidos por Santamaría (1998) para $L$. peru y se hicieron las modificaciones necesarias para la descripción de cada uno de los estadios tomando en cuenta la abundancia y el grado de desarrollo de los ovocitos y la presencia y abundancia de las células germinales. Con base en dichas descripciones se procedió a asignar el estadio que correspondiera a cada gónada.

La proporción sexual fue analizada mensualmente y por clase de talla, mediante una prueba estadística $\chi^{2}$ $(\alpha=0,05)$ para ver si existía una desviación significativa de una proporción 1:1.

El estado de desarrollo de la gónada se analizó también usando el índice gonadosomático (IGS) aplicado por Maddock \& Burton (1998) con la ecuación:

$$
\text { IGS }=\frac{\text { Peso gónada }(\mathrm{g})}{\text { Peso total }(\mathrm{g})} \times 100
$$

El factor de condición se calculo con la siguiente formula:

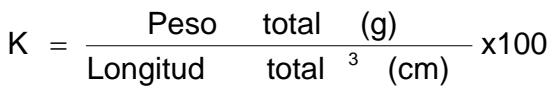

Como una medida adicional de la condición del pez, se calculó el índice hepatosomático de acuerdo con Maddock \& Burton (1998):

$$
\mathrm{IH}=\frac{\text { Peso hígado }(\mathrm{g})}{\text { Peso total }(\mathrm{g})} \times 100
$$

\section{Resultados}

\section{Proporción sexual}

Se recolectó un total de 659 especímenes adultos de los cuales 336 fueron machos (50,9\%), 323 fueron hembras $(49,1 \%)$. El rango de tallas encontrado varió entre 18 cm (que correspondió a una hembra) y 58,2 cm (que correspondió a un macho). En total la proporción de sexos fue de $1 \mathrm{M}: 0,96 \mathrm{H}$ y no se encontró una diferencia significativa $(\mathrm{P} \leq 0,05)$ de la proporción esperada de 1:1. El análisis estadístico $\left(\chi^{2}\right)$ por mes, reveló que en marzo, julio y agosto de 1993 y julio de 1994 hubo diferencias significativas en la proporción de sexos $(\mathrm{P} \leq 0,05)$ (Tabla 1). Mientras que en el análisis por clase de talla se encontró diferencia significativa $(\mathrm{P} \leq 0,05)$ en la clase de longitud de 27-30 cm (Tabla 2).

\section{Tabla 1}

Frecuencias de hembras y machos, valores de Chi cuadrado $\left(\chi^{2}\right)$ y proporción de sexos por mes de $L$. guttatus. * indica diferencia significativa de la proporción 1:1 $(\mathbf{P} \leq \mathbf{0 , 0 5})$.

Female and male frequencies, Chi square $\left(\chi^{2}\right)$ values and sex ratios of $L$. guttatus by month.

* indicate a statistically significant difference from a $1: 1$ ratio $(\mathrm{P} \leq 0.05)$.

\begin{tabular}{cccccc} 
Mes & Hembra & Macho & Total & $\chi^{2}$ & $\begin{array}{c}\text { Proporció } \\
\text { nacho: } \\
\text { Hembra }\end{array}$ \\
\hline Febrero & & & & & \\
1993 & 3 & 6 & 9 & 1 & $1: 0,5$ \\
Marzo & 8 & 19 & 27 & $4,48 *$ & $1: 0,4$ \\
Abril & 24 & 22 & 46 & 0,09 & $1: 1,09$ \\
Mayo & & & & & \\
Junio & 5 & 8 & 13 & 0,69 & $1: 0,62$ \\
Julio & 5 & 18 & 23 & $7,35 *$ & $1: 0,27$ \\
Agosto & 17 & 5 & 22 & $6,54 *$ & $1: 3,4$ \\
Septiembre & 9 & 14 & 23 & 1,09 & $1: 0,64$ \\
Octubre & 35 & 31 & 66 & 0,24 & $1: 1,12$ \\
Noviembre & 27 & 36 & 63 & 1,28 & $1: 0,75$ \\
Diciembre & 29 & 19 & 48 & 2,08 & $1: 1,5$ \\
Enero & & & & & \\
1994 & 37 & 33 & 70 & 0,23 & $1: 1,1$ \\
Febrero & 7 & 8 & 15 & 0,07 & $1: 0,8$ \\
Marzo & 9 & 17 & 26 & 2,46 & $1: 0,5$ \\
Abril & 28 & 22 & 50 & 0,72 & $1: 1,2$ \\
Mayo & 8 & 7 & 15 & 0,07 & $1: 1,1$ \\
Junio & 19 & 19 & 38 & 0 & $1: 1$ \\
Julio & 22 & 8 & 30 & $6,53 *$ & $1: 2,75$ \\
Agosto & 12 & 19 & 31 & 1,58 & $1: 0,62$ \\
Septiembre & & & & & \\
Octubre & 3 & 6 & 9 & 1 & $1: 0,5$ \\
Noviembre & 6 & 8 & 14 & 0,28 & $1: 0,7$ \\
Diciembre & 10 & 11 & 21 & 0,05 & $1: 0,9$ \\
\hline Total & 323 & 336 & 659 & 0,26 & $1: 0,96$
\end{tabular}

\section{Tabla 2}

Frecuencias de hembras y machos, valores de Chi cuadrado $\left(\chi^{2}\right)$ de $L$. guttatus por clase de longitud. El * indica diferencia significativa de la proporción 1:1 $(\mathbf{P} \leq \mathbf{0 , 0 5})$.

Female and male frequencies and Chi square $\left(\chi^{2}\right)$ values of $L$. guttatus by length class. * indicate a statistically significant difference from a 1:1 ratio $(\mathrm{P} \leq 0.05)$.

\begin{tabular}{ccccc}
$\begin{array}{c}\text { Longitud total } \\
(\mathrm{cm})\end{array}$ & Hembras & Machos & Total & $\chi^{2}$ \\
\hline $18-21$ & 24 & 29 & 53 & 0,47 \\
$21-24$ & 63 & 80 & 143 & 2,02 \\
$24-27$ & 90 & 114 & 204 & 2,82 \\
$27-30$ & 57 & 35 & 92 & $5,26^{*}$ \\
$30-33$ & 39 & 32 & 71 & 0,69 \\
$33-36$ & 19 & 19 & 38 & 0
\end{tabular}


Tabla 2 (continuación)

\begin{tabular}{ccccc}
$\begin{array}{c}\text { Longitud total } \\
(\mathrm{cm})\end{array}$ & Hembras & Machos & Total & $\chi^{2}$ \\
\hline $36-39$ & 15 & 9 & 24 & 1,5 \\
$39-42$ & 6 & 7 & 13 & 0,08 \\
$42-45$ & 4 & 4 & 8 & 0 \\
$45-48$ & 0 & 2 & 2 & 2 \\
$48-51$ & 2 & 0 & 2 & 2 \\
$51-54$ & 0 & 0 & 0 & \\
$54-57$ & 4 & 2 & 6 & 0,67 \\
$57-60$ & 0 & 3 & 3 & 3 \\
\hline Total & 323 & 336 & 659 & 0,26
\end{tabular}

\section{Descripción microscópica de los estadios de desarrollo gonadal}

Se definieron 5 estadios (en reposo, en desarrollo, maduro, en desove/eyaculación y en posdesove / poseyaculación) tanto para hembras como para machos.

Ovario:

En reposo: Se caracteriza por que las lamelas ovígeras, en las que se encuentra el epitelio germinal, son claramente visibles. Se presentan exclusivamente ovogonias, ovocitos tempranos y previtelogénicos. El lumen es generalmente grande.

En desarrollo: La separación entre las lamelas ovígeras ha disminuido por lo que no son tan evidentes. Además de ovocitos tempranos y previtelogénicos se presentan ovocitos en vitelogénesis inicial. En fases avanzadas de desarrollo se presentan también ovocitos en vitelogénesis avanzada.

Maduro: No hay una separación evidente entre las lamelas ovígeras. Los ovocitos previtelogénicos y vitelogénicos son menos evidentes. Abundan los ovocitos en vitelogénesis avanzada y en fases avanzadas de madurez se presentan algunos ovocitos prehidratados e hidratados.

En desove: Se caracteriza por la presencia de folículos postovulatorios, en diferentes grados de reabsorción (evidenciando la expulsión de gametos), y de ovocitos hidratados. Los ovocitos tempranos y previtelogénicos empiezan a ser más evidentes.

En postdesove: Se caracteriza por la presencia de atresias de ovocitos en vitelogénesis avanzada en diferentes grados de reabsorción y por presentar espacios vacios en el tejido gonadal. Sólo se presentan ovocitos tempranos y previtelogénicos.

Testículo:

En reposo: Se observa escasa actividad espermatogénica, en los cistos predominan las espermatogonias y espermatocitos I y II. Se pueden llegar a observar algunos espermatozoides residuales. El testículo tiene una apariencia compacta.

En desarrollo: Se presenta gran actividad espermatogénica. En los cistos se observan células espermatogénicas en todas las etapas de desarrollo (espermatogonias, espermatocitos I y II, espermatogonias y espermatozoides)

Maduro: En la parte central el lumen se observa ensanchado y lleno de espermatozoides, se presenta escasa actividad espermatogénica en los cistos de la periferia.

En eyaculación: Se aprecian espacios vacíos, los espermatozoides se encuentran en mayor cantidad en la parte central del testículo. Se presenta escasa actividad espermatogénica en los cistos de la periferia.

Poseyaculación: La actividad espermatogénica en los cistos es casi nula. Se observan gran cantidad de espacios vacíos dentro de los túbulos pero existen espermatozoides residuales.

\section{Ciclo reproductivo}

La presencia de varios grupos modales de ovocitos intraováricos en diferentes fases de desarrollo y folículos postovulatorios en diferente grado de reabsorción, revelaron que esta especie presenta un desarrollo gonadal asincrónico de la gónada y desoves parciales. El testículo es de tipo tubular, la distribución de las espermatogonias es del tipo no restringido y se distinguen cistos en los cuales el desarrollo espermatogénico es sincrónico.

En la Figura 2a y 2b se presentan las frecuencias de ocurrencia de las diferentes fases de desarrollo gonadal para hembras y machos a lo largo de los dos años en que se realizó el estudio. Se puede observar que $L$. guttatus presenta gónadas en desarrollo, maduras y en desove/eyaculación durante la mayor parte del año lo que indica que esta especie tiene un período reproductivo prolongado. Sin embargo, se pueden apreciar dos picos reproductivos (más del 50\% de los especímenes maduros): el primero entre marzo y abril y el segundo de agosto a noviembre con el máximo de gónadas maduras en agosto 1994 (87.5 \%). Las gónadas en desove/eyaculación se presentaron a lo largo de casi todo el ciclo anual, pero con porcentajes bajos para ambos sexos, entre $11.1 \%$ (marzo 1994) y 33,3 \% (agosto 1994).

\section{Índices gonadosomático, hepatosomático y factor de condición.}

Los valores medios mensuales del IGS presentan gran variación a lo largo del período de estudio en ambos sexos y aunque los valores son claramente mayores en el caso de las hembras, se observan tendencias similares en ambos sexos. Se detectaron dos picos por año: en marzo y en 


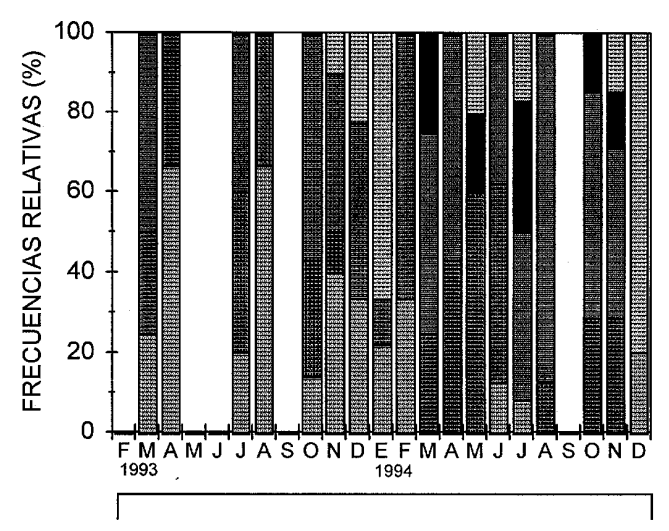

a

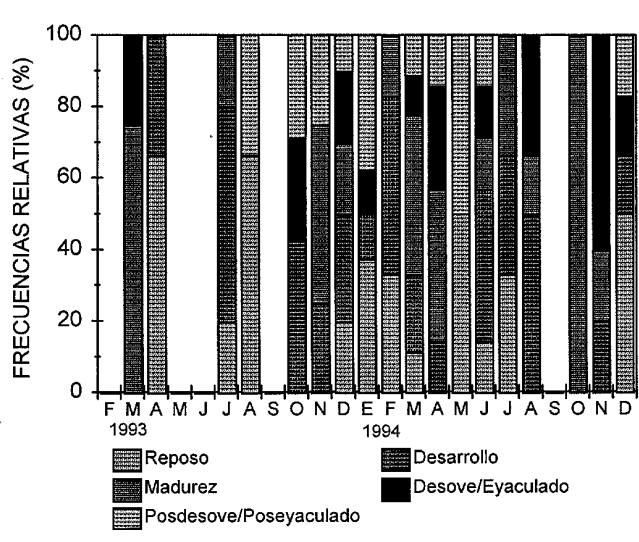

Figura 2

Ciclo reproductivo de (a) hembras y (b) machos de $L$. guttatus. Frecuencias relativas de las fases de desarrollo gonadal entre febrero de 1993 y diciembre de 1994.

Reproductive cycle of (a) females and (b) males of $L$. guttatus. Relative frequency of gonadal stages between February 1993 and December 1994.

octubre-diciembre en 1993, mientras que en 1994 en marzo-abril y en agosto-noviembre (Fig. 3a). Los valores promedios más altos del IGS en ambos sexos coinciden con las frecuencias más altas de organismos maduros analizados con los resultados histológicos.

En el caso del índice hepatosomático, se observa que los valores medios mensuales presentan una gran variación a lo largo del período de estudio y siguen las mismas tendencias en ambos sexos. A pesar de que no se observa una clara relación directa o inversa entre los valores del IGS y del IH, sí es posible notar que en los meses donde se observan los picos del IGS los valores del IH son bajos, excepto en octubre de 1994 (Fig. 3b).

El factor de condición presenta valores altos en el mes de marzo en el caso de las hembras y en febrero y abril en el caso de los machos, coincidiendo con el primer máximo reproductivo de la especie de acuerdo con los valores del IGS y con los resultados histológicos. Sin embargo, se observa una tendencia del FC a disminuir conforme transcurre el ciclo reproductivo (Fig. 4).
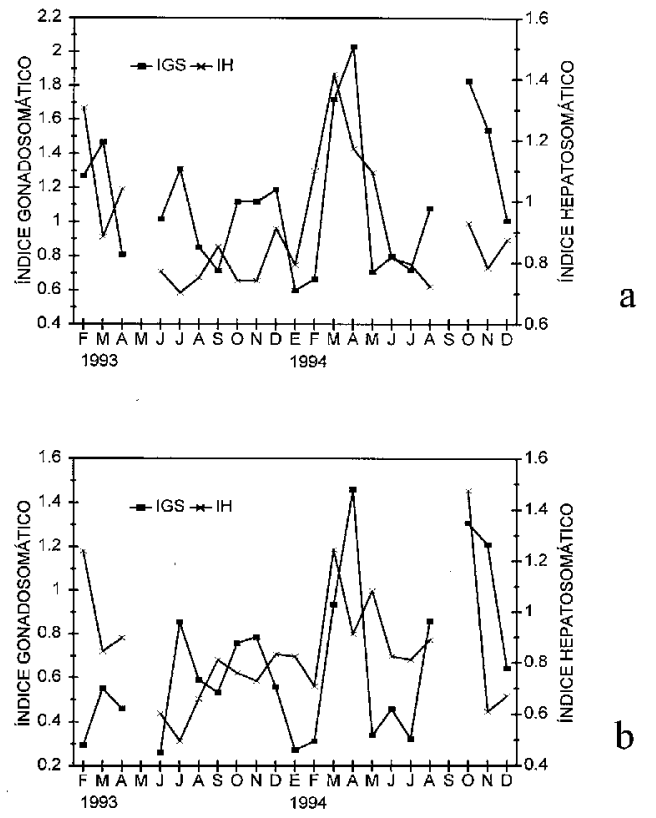

b

Figura 3

Variación mensual de los valores medios del índice gonadosomático y del índice hepatosomático de $L$. guttatus de las costas de Guerrero, México. a) hembra, b) macho.

Monthly variation of the mean values of gonadosomatic index and hepatosomatic index of $L$. guttatus from coast of Guerrero, Mexico. a) female, b) male.
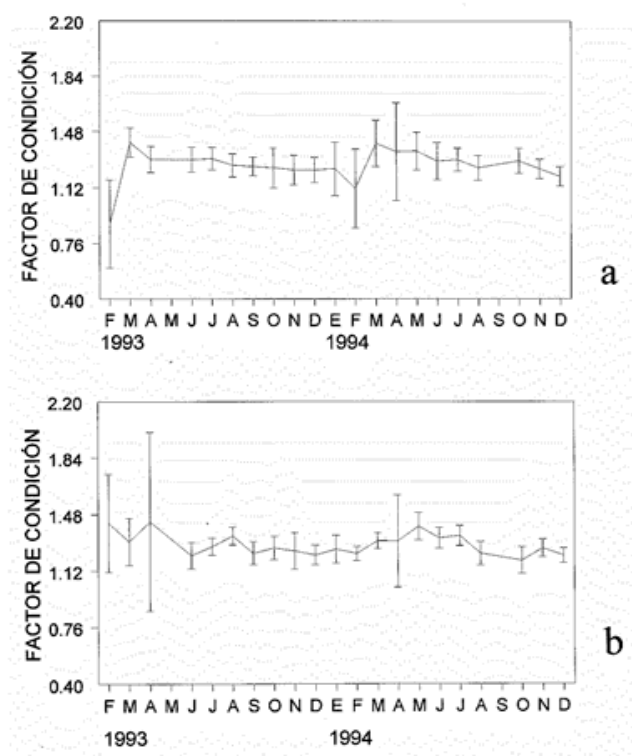

Figura 4

Variación mensual de los valores medios del factor de condición de $L$. guttatus de las costas de Guerrero, México. a) hembra, b) macho.

Monthly variation of the mean values of condition factor of $L$. guttatus from coast of Guerrero, Mexico. a) female, b) male. 


\section{Discusión}

La proporción de sexos del pargo lunarejo L. guttatus para el total de la muestra es de 1 macho a 0.96 hembras pero no se encontró diferencia significativa de la proporción esperada de 1:1, como lo demuestra el análisis estadístico. Rojas (1997) reporta que no encontró diferencia significativa en la proporción de sexos para la misma especie en el Golfo de Nicoya, Costa Rica. Sin embargo, el análisis estadístico reveló en este estudio que en algunos meses sí existe una diferencia significativa. Estas diferencias en la proporción de sexos han sido atribuidas, entre otros factores, a una distribución diferencial de una fracción de la población (Moyer \& Nakazono 1978, Moyer et al. 1983, Aranis \& Torres 1987). Starck (1971) reportó que los machos de $L$. griseus, en Florida, generalmente son más abundantes en las zonas arrecifales, lejos de la costa, mientras que las hembras son más abundantes en la zona costera, debido a que se acercan para desovar, por lo que están más expuestas a las artes de pesca, aunque Claro (1994) encontró que la proporción sexual de los pargos tiende a igualarse durante el desove. Rojas (1997) encontró para L. guttatus una proporción sexual de 1,3 machos por cada hembra en el Golfo de Nicoya, Costa Rica y atribuye que esta proporción se debe posiblemente a un comportamiento gregario en un área que podría estar relacionada con la distribución y demanda de alimento, más que por algún comportamiento social.

L. guttatus es una especie con sexos separados (gonocórica) que presenta los tipos de células gonadales (Wallace \& Selman 1981, De Vlaming 1983, Guraya 1986) y la morfología funcional de las gónadas (Nagahama 1983) comunes en los teleósteos.

Como el análisis histológico de las gónadas reveló la presencia de varias tallas modales de ovocitos y en diferentes fases de madurez simultáneamente, se demuestra que $L$. guttatus presenta un desarrollo gonadal asincrónico. Adicionalmente, se encontró evidencia de que $L$. guttatus presenta desoves parciales pues se encontraron ovarios con ovocitos con un grado de desarrollo avanzado y folículos postovulatorios en distinto grado de reabsorción. Esto último sugiere que los ovocitos fueron liberados a diferentes tiempos.

En ésta y en otras especies de Lutjanus se ha observado esta misma característica (Claro 1983, Rojas 1997, Santamaría 1998). La organización celular de los testículos de L. guttatus es como la de la mayoría de los teleósteos presentando espermatogonias a todo lo largo de los tubulos (Grier 1981). Asimismo, el desarrollo espermatogénico dentro de los cistos es sincrónico, característica común en teleósteos (Grier 1981).

Del análisis del ciclo reproductivo y como se encontraron organismos maduros y/o en desove durante todo el año, se pudo determinar que $L$. gutattus se reproduce todo el año con 2 máximos reproductivos: marzo de 1993 y marzo-abril de 1994 y uno más prolongado que va de agosto a noviembre en el caso de las hembras y hasta diciembre en el caso de los machos, tal como lo menciona Cruz-Romero et al. (1988) para la misma especie en el estado de Colima, México. Rojas (1997) encontró que esta especie se reproduce todo el año en Costa Rica con 2 máximos reproductivos (abril y octubre).

Existe una relación entre la duración de la temporada de reproducción, el tipo de desove y la latitud (Cushing 1975, Blaxter \& Hunter 1982). Así, en las especies de latitudes altas, donde el período estival es corto, se observa que las especies presentan generalmente un período de desove corto y definido y un tipo de desove total (Cushing 1975, Blaxter \& Hunter 1982). A medida que se avanza hacia el Ecuador, es decir en latitudes correspondientes a las zonas subtropical y tropical, el período de reproducción de los peces es más prolongado, puede limitarse a una temporada más amplia pero definida con desoves parciales o puede durar todo el año (Cushing 1975). Este último es el caso de L. guttatus. Así mismo, para otras especies de la familia Lutjanidae de zonas subtropicales y tropicales, tanto del Océano Pacífico como del Atlántico, se han reportado períodos prolongados de actividad reproductiva (Munro et al. 1973, Thomson \& Munro 1973, Brouard \& GranPerrin 1984, Cruz-Romero et al. 1991, Santamaría 1998).

Los valores medios mensuales del IGS no presentan una tendencia clara, por el contrario, presentan gran variación a lo largo del período de estudio. Esto puede deberse a que hay actividad reproductiva todo el año y no toda la población se reproduce al mismo tiempo dando como consecuencia que cada mes se presente más de un estadio de madurez (por lo tanto gónadas de diferentes pesos) y con ello se produzca la gran variación en los valores del IGS.

Sin embargo, los valores máximos del IGS (marzo, agosto-noviembre) coinciden con los períodos máximos reproductivos establecidos histológicamente. Por lo tanto, se puede considerar que el IGS es predictivo de los principales picos de actividad reproductiva.

Tradicionalmente se usa el FC para comparar la condición fisiológica y la condición reproductiva de peces sin embargo, en $L$. guttatus el IGS y el FC presentan la misma tendencia. Por lo tanto se utilizó el IH, el cual se considera que en peces también es un buen indicador de la condición fisiológica (Tyler \& Dunn 1976) y encontramos una mejor evidencia de una posible transferencia energética durante el proceso reproductivo. En $L$. guttatus, los valores altos del IH durante los períodos de inactividad reproductiva pueden ser producidos por la acumulación de sustancias de reserva, para ser usado 
durante la maduración. La presencia de valores altos del IH durante el desarrollo gonadal y valores bajos antes del máximo desarrollo gonadal y durante el desove, pueden ser interpretados como una baja en las reservas energéticas almacenadas en el hígado en forma de glucógeno, el cual es transferido a la gónada para ser usado en el proceso reproductivo, produciendo una baja en la condición de los peces durante el período de desove (Bagenal 1957, Rajasilta et al. 1997, Ceballos-Vázquez 1993, Maddock \& Burton 1998). La disminución en el factor de condición conforme transcurre el ciclo reproductivo confirma lo anterior y se explica por el hecho de que la actividad reproductiva implica un alto costo energético para los organismos.

A pesar de no encontrar una relación clara entre el IGS y el IH de ambos sexos, sí se encontró que en los meses de máximo valor del IGS, los valores del IH son bajos. Por lo tanto se puede sospechar que existe una relación inversa entre la actividad reproductiva y la condición fisiológica del pez. Sin embargo es necesario realizar estudios más detallados y específicos que permitan precisar la interacción de la actividad reproductiva con la condición fisiológica del pargo lunarejo.

\section{Agradecimientos}

Se agradece a la Escuela Superior de Ecología Marina por el aporte económico para llevar a cabo esta investigación. Al personal de esa escuela los cuales dieron el apoyo logístico para la recolección de las muestras.

\section{Literatura Citada}

Allen GR. 1985. Snappers of the world. An annoted and illustrated catalogue of lutjanid species known to date. FAO Fish. Synopsis, 208 pp.

Allen GR. 1995. Lutjanidae. Pp. 1231-1244. En: Fischer W., F. Krupp, W. Schneider, V.H. Niem (Red. Téc.). Guía FAO para la identificación de especies para los fines de la pesca. Vol. III Pacífico Centro-oriental. FAO, CE, FIS, NORAD. Roma.

Aranis R. \& A Torres. 1987. Un caso de hermafroditismo en la sardina (Sardinops sagax Jenyns, 1842) (Pisces, Clupeiformes, Clupeidae). Investigación Pesquera 34: 149-153.

Bagenal TB. 1957. The breeding and fecundity of the long rough dab Hippoglossoides platessoides (Fabr.) and the associated cycle in condition. Journal of Marine Biology Association. U. K. 36: 339-375.

Brouard W \& R GrandPerrin. 1984. Les poissons profonds de la pinta recifale externe a Vanatu. Notes Documentaires D' Oceanography 11: 71-79.

Blaxter JHS \& JR Hunter. 1982. The biology of the clupeoid fishes. Advances in Marine Biology 20: 1-223.
Ceballos-Vázquez BP. 1993. Biología reproductiva del conejo Caulolatilus affinis, Gill 1865 (Pisces: Branchiostegidae), en el canal Cerralvo, Baja California Sur, México. Tesis de maestría CICIMAR-IPN, La Paz, B.C.S. México

Claro R. 1983. Ecología y ciclo de vida del caballerote Lutjanus griseus (Linnaeus), en la plataforma cubana. Identidad, distribución y hábitat, alimentación y reproducción. Reporte Oceanológico. Academia de Ciencias de Cuba 7:1-30.

Claro R. 1994. Ecología de los peces marinos de Cuba. Instituto de Oceanología Academia de Ciencias de Cuba y Centro de Investigaciones de Quintana Roo, México. 525 pp.

Cruz-Romero M, E Espinoza-Bar, J Mimbela-López, A García-Boa, L Obregón-Alcaráz \& E Girón-Botello. 1988. Aspectos biológico-pesqueros de tres especies de Lutjánidos en Colima, México. Reporte técnico del CRIP de Manzanillo, Colima. I.N.P. 31 pp.

Cruz-Romero M, E Espinoza-Bar, J Mimbela-López, A García-Boa, L Obregón-Alcaráz \& E Girón-Botello. 1991. Biología reproductiva de tres especies del género Lutjanus en las costas de Colima, México. Reporte de investigación del CRIP de Manzanillo, Colima. Instituto Nacional de Pesca. 118 pp.

Cushing DH. 1975. Ecología marina y pesquerías. Editorial Acribia, 252 pp.

De Vlaming V. 1983. Oocyte developmental patterns and hormonal involvements among teleosts. En: Rankin J.C., T.J. Pitcher \& R.T. Duggan (Eds.) Control Processes in Fish Physiology. 99-176. Croom Helm, Londres.

Guraya, SS. 1986. The cell and molecular biology of fish oogenesis (Karger: Basel. En: West, G. 1990. Methods of assesing ovarian development in fishes: A review. Australian Journal of Marine and Freshwater Research 41: 199-222.

Humason GL. 1979. Animal Tissue Techniques. 4th edition. W. H. Freeman and Co. San Francisco, 692 pp.

Maddock DM \& MP Burton. 1998. Gross and histological of ovarian development and related condition changes in American plaice. Journal of Fish Biology 53: 928-944.

Moyer JT \& A Nakazono. 1978. Population structure reproductive behaviour and protogynous hermaphroditism in the angelfish Centropyge interruptus at Miyake-jima, Japan. Japanese Journal of Ichthyology 25: 25-39.

Moyer JT, RE Thresher \& PL Colin. 1983. Courtship, spawning and inferred social organization of American angel fish (Genera Pomacanthus, Holacanthus and Centropyge; Pomacanthidae). Environmental Biology of Fishes 9(1): 25-39.

Munro J, V Gaut, $R$ Thomson \& $P$ Reeson. 1973. The spawning seasons of Caribbean reef fish. Journal of Fish Biology 5:69-84.

Nagahama Y. 1983. The functional morphology of teleost gonads. 223-275 pp. En: Fish Physiology. Vol. IX: Reproduction. Hoar W.S., D.J. Randall \& E.M. Donaldson (Ed). Academic Press. Nueva York. 
Rajasilta M, J Paranko \& T Laine. 1997. Reproductive characteristics of the male herring in the northern Baltic Sea. Journal of Fish Biology 51: 978-988.

Rojas MJR. 1997. Fecundidad y épocas de reproducción del "pargo mancha" Lutjanus guttatus (Pisces: Lutjanidae) en el Golfo de Nicoya, Costa Rica. Revista de Biología Tropical 44(3): 477-487.

Santamaría MA. 1998. Hábitos alimenticios y ciclo reproductivo del huachinango, Lutjanus peru (Nichols y Murphy, 1922) Pisces: Lutjanidae en Guerrero, México. Tesis de maestría, Centro Interdisciplinario de Ciencias Marinas, La Paz, B.C.S. México, 64 pp.

Starck WA. 1971. Biology of gray snapper, Lutjanus griseus (Linaeus) in the Florida Keys. Studies in Oceanography 10: 11-150.
Thomson R \& L Munro. 1973. The biology, ecology and bionomics of the snappers, Lutjanidae. 94-109. En: J.R.Munro (Ed.). Caribbean coral reef fishery resources. ICLARM, Indian Occidentales.

Tyler AV \& RS Dunn. 1976. Ration, growth and measures of somatic and organ condition in relation to meal frequency in winter flounder, Pseudopleuronecte americanus, with hypotheses regarding population homeostasis. Journal of the Fisheries Research Board of Canada 33: 63-75.

Wallace RA \& K Selman. 1981. Cellular and dynamic aspects of oocyte growth in teleosts. American Zoologist 21: 325-343. En: West, G. 1990. Methods of assesing ovarian development in fishes: A review. Australian Journal of Marine and Freshwater Research 41: 199-222. 